\title{
Pablo Latapí (1926-2009). Educador de Iberoamérica*
}

\section{Pablo Latapí (1926-2009). Ilbero-American Educator}

Sylvia Schmelkes ${ }^{* *}$

\section{PALABRA DE ADVERTENCIA}

Tuve el privilegio de ser formada por Pablo Latapí, de haberlo conocido durante 40 años, de haber tenido con él una profunda amistad. Conozco su vida y su obra. No puedo resumirlo en 30 minutos de exposición. He tenido que ser selectiva en lo que aquí presento. He procurado hacer un esfuerzo de abstracción y síntesis, pero dudando de mi objetividad, he descansado en los escritos del propio Pablo Latapí, sobre todo los últimos, donde él mismo realiza un recuento de su vida.

26. Andante con Brio (2008) trata de la relación entre la investigación y la política educativa durante más de cuatro décadas, a través de sus interacciones con los secretarios de educación. Es una selección que permite que emerja toda la fuerza de la persona de su autor, sus consistentes búsquedas, su ejemplar rectitud, sus valores inquebrantables y su propia evolución. Presenta una historia de 40 años de educación en México desde el sitio en que le tocó estar: como crítico externo, colaborador formal de quienes tomaban las decisiones, colaborador informal y más esporádico y, siempre, como pensador independiente.

27. Poco antes de saber que estaba enfermo, regaló a sus amigos un libro impreso en edición limitada de 200 ejemplares, en el que seleccionó sus trabajos pequeños que más le gustaban. Tituló su libro Porque ya Atardece. Su legado más preciado,

*Participación en el Panel "La Construcción de una Pedagogía en Iberoamérica: los Grandes Pedagogos del Siglo XX”, en el marco del Congreso Iberoamericano de Pedagogía, 2018, celebrado en Buenos Aires, Argentina, del 14 al 17 de agosto de 2018.

"Consejera de la Junta de Gobierno del Instituto Nacional para la Evaluación de la Educación de México; schmelkes@gmail.com 
dedicado a mí y a mi esposo: "Para Sylvia y Pepe, que reconocerán su propio proyecto en el mío, que relatan estas páginas".

Después de saber de su cáncer terminal, que enfrentó con una fe envidiable, traducida, para nosotros, en una paz incomprensible que logró transmitirnos, se dio a la tarea de preparar dos libros más: Finale Prestissimo. Pensamientos, vivencias y testimonios, editado por el Fondo de Cultura Económica (FCE), y una antología de sus escritos sobre educación de adultos, que publicó el Centro de Cooperación Regional para la Educación de Adultos en América Latina y el Caribe (CREFAL). Estas últimas dos entregas a la comunidad de educadores, la actual y la futura, lo mantuvieron con vida y entusiasmo durante sus últimos siete meses. Dos semanas después de terminar sus libros, murió.

Me queda la sensación de dejar mucho, demasiado, fuera. Por eso tomen lo que diré a continuación como una invitación a leer y a conocer su obra, a hacerla objeto de estudio, y a convertirla en referencia obligada de los futuros pedagogos, educadores e investigadores educativos de Iberoamérica. Lo considero indispensable.

\section{PADRE DE LA INVESTIGACIÓN EDUCATIVA EN IBEROAMÉRICA}

Es reconocido así en México. Pero lo es también al menos en América Latina, pues promovió también la creación de los primeros centros de investigación educativa en varios países de la región.

Convencido desde joven de que la educación tenía un potencial transformador profundo de personas y de sociedades, trazó su plan de vida, siendo entonces un joven jesuita -más tarde dejaría la orden-, para especializarse en este tema. Terminados sus estudios de filosofía y teología, consigue permiso para ir a estudiar un doctorado en filosofía de la educación en Hamburgo. $\mathrm{Su}$ estancia en Europa le permite vincularse con la investigación educativa que allá se realizaba. Visitaba con frecuencia los diferentes organismos de la UNESCO y ahí asistía a conferencias, trabajaba en las bibliotecas, conversaba con personajes de diversas 
partes del mundo. Cuando volvió a México, formado como Doctor en Educación y con esa visión internacional actualizada tanto de la problemática educativa como de las maneras de abordarla, emprende la tarea de crear lo que sería el primer centro de investigación educativa en el país: el Centro de Estudios Educativos (CEE), en 1963. Volvió convencido de que la educación es un campo del conocimiento en el que confluyen muchas disciplinas: la filosofía, la antropología, la psicología, la sociología, la economía. Con la capacidad de visión que lo caracterizó siempre, y comenzando con un muy pequeño grupo de investigadores jóvenes, crea un centro de investigación que es justamente eso: interdisciplinario, décadas antes de que la interdisciplinariedad se convirtiera en búsqueda explícita en nuestras instituciones de educación superior.

Su capacidad de pensar a futuro lo lleva a enviar a estos investigadores, en forma escalonada, a estudiar posgrados fuera del país. De esta manera, constituye un núcleo de personas de primer nivel que van a hacer del CEE una referencia obligada para la educación en el país y más allá de él. Con los años, se crean varios centros en América Latina a su imagen y semejanza.

Pero, además, como padre de la investigación educativa, creó escuela, tiene discípulos, marca la impronta de arranque, deja la huella genética de su origen. También sigue formando a las nuevas generaciones, porque sus trabajos son objeto de estudio obligado en todas las escuelas de educación en México, y probablemente lo sean también en toda Iberoamérica.

\section{LO FUNDAMENTAL DE SU PERSONA Y SU OBRA}

\section{Es ante todo un investigador de la educación}

Cuando Pablo crea el CEE, solamente existía el Centro de Investigaciones Pedagógicas, de la SEP, que elaboraba, fundamentalmente, pruebas psicométricas. Su perspectiva fue interdisciplinaria. Diecisiete años después, cuando dirige el Programa Nacional Indicativo de Investigación Educativa del Consejo Nacional de Ciencia y Tecnología (Conacyt), realiza un inventario de 200 instituciones o centros dentro de instituciones de educación su- 
perior en el país que dicen hacer investigación educativa. Entre éstos selecciona 20 que, sin duda, la realizan. En poco tiempo la investigación educativa estaba instalada en el panorama nacional.

No solamente sembró la idea de la importancia de la investigación educativa, sino que nos enseñó cómo hacerla. Destaco tres aspectos fuertes:

- El quehacer de la investigación educativa implica generar conocimiento científico, y hacerlo requiere rigor.

- El quehacer de la investigación educativa no implica neutralidad valoral sino, por el contrario, supone una ética de compromiso con la justicia educativa y social. Nos enseńó que el rigor científico implica objetividad, pero no neutralidad.

- Que investigamos para transformar la realidad, y que para hacerlo es indispensable divulgar ampliamente el conocimiento generado y las propuestas de política que de ahí se derivan. Por eso Pablo mantuvo con enorme disciplina su rica actividad periodística, en la que lo coyuntural servía para realizar reflexiones filosóficas y políticas profundas. "La IE no es una mera técnica, sino un ejercicio que se integra en una visión holística de lo que somos como seres humanos", dice en Finale Prestissimo.

Pablo Latapí fue un científico. Valoró el rigor académico, el andamiaje teórico y metodológico indispensable para que la búsqueda de la verdad en el campo de la educación sea certera y tenga credibilidad. Fue un maestro del rigor. Inició la investigación educativa en México poniendo estándares elevados de precisión y honestidad intelectual. Son estándares que hasta la fecha se utilizan para juzgar la calidad de las investigaciones educativas y han dado a nuestro país una gran fortaleza en este campo del conocimiento. Una buena parte de la investigación educativa que se realiza en México es, en gran parte, debido a él, investigación de calidad.

Su trabajo trascendió el país. Latapí tiene un reconocimiento internacional innegable. La fortaleza de este reconocimiento procede de su creciente dominio de la problemática social y educativa de nuestro subcontinente, y de sus certeras propuestas para su transformación. 


\section{Su compromiso}

Pablo Latapí nunca investiga por investigar; no persigue generar conocimiento por el conocimiento mismo. Siempre hay una motivación transformadora detrás de lo que investiga, de lo que escribe. Sus trabajos tienen claramente una orientación política: se trata de recomendar para transformar. La transformación siempre está referida al logro de una mayor justicia social, que muchas veces depende de la calidad con la que se ofrece, pero también de la equidad con la que se distribuye. Sus trabajos de investigación siempre están motivados por la existencia de una problemática, y abonan, siempre, a su solución.

Informes de investigaciones. Artículos periodísticos. Producción ensayística vasta. Lo importante para él fue asegurar que, lo que valía la pena decir, llegara a quienes toman decisiones y a la sociedad en general. Siempre sostuvo que una sociedad bien informada tendría mucha mayor capacidad de influir certeramente en la toma de decisiones; su convicción era que esto constituía un ejercicio profundamente democrático.

Esta característica del compromiso que Pablo Latapí asignó a su vida y a su obra nos muestra, con claridad, que un científico tiene que ser riguroso, sí, y objetivo también, pero que eso no implica que deba ser neutral. El es testimonio de que un científico de la educación, por la temática que trabaja, debe tener un compromiso con la sociedad en la que vive, y debe procurar siempre que la educación, al estudiarla, pueda ofrecer algo mejor a las personas y, con ello, aportar a la transformación de la sociedad. Es éste otro estándar que puso a la investigación educativa (Schmelkes, 2000).

\section{Su humanismo}

Siempre presente en sus escritos, pero de forma más patente en los últimos. Como el maestro que fue, nos enseña que no se puede ser educador ni investigador educativo, que no se puede trabajar en educación sin tener a la persona humana como referente central de lo que hacemos. La persona, siempre en contexto, es lo que da sentido a lo que hacemos todos y cada uno de los que 
trabajamos en educación. Pablo Latapí parte de la premisa de que los seres humanos son diversos y de que ello es una fortuna. Pero también está convencido de su dignidad inherente: el derecho a crecer, a desarrollar su potencial y a disfrutar de una vida de calidad, como cada quien la defina, desde su contexto, es el propósito del hecho educativo.

Pablo Latapí no soportaba que las personas sufrieran ni que se obstaculizara su desarrollo. Desde que inició su trabajo, tuvo claro que el hecho de que esto ocurriera era producto de una historia de dominación y opresión, así como de estructuras sociales injustas. Visualizó desde muy temprano que una buena educación, bien distribuida, podría, junto con otros procesos, ir transformando estas estructuras. A la vez, y paradójicamente, tenía claro que era necesario que estas estructuras se transformaran para poder dar lugar al desarrollo de todas las personas. Las propuestas de una sociedad distinta, como la manifiesta en la filosofía del movimiento zapatista de 1994, lo capturaron profundamente. Su preocupación central por la justicia, que él mismo define y muchos reconocen como lo que marca su vida y su obra, deriva de esta manera de entender el problemático binomio ser humano-sociedad.

Como buen educador, valora de manera especial el trabajo de los docentes y de los agentes educativos. Reconoce su trabajo comprometido, constante, silencioso. Sabe que de su esfuerzo cotidiano depende el hecho educativo y por eso los aprecia. La persona del educando, y la del docente, como binomio educativo esencial, son el centro de su pensamiento educativo humanista.

Otras características de su persona explican también la potencia de su obra. Dominó el idioma y su escritura. Su formación retórica hace de cada pieza una obra maestra en composición. Es persuasivo por la contundencia de sus argumentos. Es sencillo y claro, de manera que logra que muchos lo entiendan sin problema y que, después de leerlo, no puedan sino decir: "tiene razón”. Es crítico siempre, e incisivo en su crítica. La crítica se transforma en propuesta en la gran mayoría de sus escritos, pero lo más asombroso de su estilo es su capacidad de provocar la reflexión. Siempre es posible dialogar con él como escritor. Y cada uno de sus escritos tiene la capacidad de remover certezas y de dejar pensando. 


\section{CREADOR DE INSTITUCIONES Y FORMADOR DE INVESTIGADORES}

Como toda persona grande, que trasciende, Pablo fue innovador $\mathrm{y}$ creador de instituciones, que a su vez formaron personas en las capacidades y en las causas que cada institución perseguía. Ya mencionamos al Centro de Estudios Educativos, que este año cumple 55 años de existencia. En su seno nace, a instancias de Pablo como su entonces director, la Revista Latinoamericana de Estudios Educativos, órgano de alto nivel con vocación regional, que dio a la luz los primeros estudios serios con enfoque social y económico sobre la educación en América Latina. La revista cumplió, en 2018, 48 años de existencia.

Su interés por los asuntos de la educación superior lo llevó a crear la asociación civil no lucrativa denominada Prospectiva Universitaria, dedicada a estudiar el futuro de las universidades en el mundo y en América Latina. Más o menos por estas fechas creó las Reuniones de Información Educativa, eventos periódicos que reunían a investigadores y tomadores de decisiones en torno a temas que eran objeto de hallazgos relevantes para la política educativa.

En 1985 fundó la Asociación Mexicana para las Naciones Unidas. Esta asociación existe en varios países para defender, desde los pueblos, las causas de la ONU.

Hacia el año 2000 convence a un grupo de académicos para crear el Observatorio Ciudadano de la Educación, organización pequeña, integrada en su mayoría por voluntarios que se propusieron como objetivo dar a conocer a la opinión pública, mediante comunicados de prensa, lo que la investigación educativa informaba a los tomadores de decisiones en educación, para orientar la política educativa y también la práctica escolar y docente. En sus poco más de 10 años de existencia emitió alrededor de 200 comunicados y organizó cerca de 20 foros sobre temáticas muy diversas. Su portal llegó a recibir cerca de 20000 visitas al año. Todavía pueden consultarse y son documentos históricos de gran valor. 


\section{LA INVESTIGACIÓN Y LA POLÍTICA EDUCATIVA}

Pablo Latapí siempre consideró la investigación educativa como el instrumento para hacer su contribución a la tarea utópica de construir una sociedad más justa. La confianza en su potencia lo mantuvo siempre firme en su vocación. Esto nos explica en buena parte de sus memorias, dedicadas a la reflexión profunda y minuciosa sobre la relación entre la investigación y la toma de decisiones en materia educativa. Destaca su comprobación de la tesis de Carlos Muñoz Izquierdo respecto a esta relación, en el sentido de que es el proceso político el que determina que se adopte una innovación cuya necesidad y fundamento ( $\mathrm{y}$ a veces incluso prueba previa) procede de la investigación educativa. Las condiciones políticas tienen que estar dadas para que esto ocurra.

Relata cómo esto fue posible en torno a tres de los temas fundamentales de su consistente preocupación: el primero es el de la equidad educativa, que pudo favorecer con Fernando Solana como secretario de Educación a través de un programa que se denominó Primaria para Todos los Niños, que Pablo ayudó a diseñar, así como con Ernesto Zedillo, con el capítulo de Equidad en la Ley General de Educación de 1993. El segundo tema es la formación en valores. El tercero es la participación social en la educación, también incluida en la Ley General de Educación de 1993.

Pablo Latapí añade a la tesis de Carlos Muñoz el tema de las mediaciones necesarias para que, dada la coyuntura favorable, la influencia se logre. Una de ellas es el papel de un asesor, como él lo fue: investigador de la educación y conocedor de la misma, de sus contextos y de su historia; crítico y a la vez propositivo; generalista, no especialista; conocedor de las restricciones de los políticos y capaz de construir propuestas realizables -lo que él llama "la sensatez necesaria" de quien propone transformaciones-. Requisito de esta mediación es el mutuo respeto y aprecio, la empatía, y sobre todo, la convergencia de valores esenciales entre asesor y asesorado.

Otra mediación que Pablo desarrolla es la externa, mucho más lenta y compleja: la de la formación de opinión pública, que conduce a la participación social en educación y permite que una 
decisión de política resulte políticamente necesaria. Una labor que Pablo realizó a través de su trabajo periodístico y de la creación de instituciones, que con el tiempo va forjando democracia educativa.

Pero una conclusión que el lector saca de la lectura de este libro es que nada de lo que ahí se relata hubiera sido posible si no se tratara de una persona de la estatura de Pablo Latapí. Su integridad valoral lo mantuvo siempre independiente. Sin interrupción, mostró su pensar y sentir en la prensa. Rechazó la invitación de un secretario cuestionado por haber intervenido en los resultados de las elecciones "porque este gobierno carece de legitimidad", y renunció a su asesoría con otro más a raíz de la masacre de Acteal, por no soportar la incongruencia de trabajar por un régimen que la había permitido.

En sus reflexiones analiza las causas de fondo de la falta de calidad educativa. El hecho de que la educación no ha sido, para ningún gobierno federal, verdadera prioridad, que tampoco lo ha sido para los legisladores; que los empresarios auténticamente preocupados se cuentan con los dedos de una mano; que el magisterio es prisionero del Sindicato, y que la ciudadanía es en exceso complaciente respecto del servicio educativo recibido. Plantea la necesidad de avanzar en democracia y en participación social en materia de educación. Sostiene que mientras la sociedad no crezca en su capacidad de exigir cuentas al gobierno, a los legisladores, a los sindicatos, la mala calidad y la desigual distribución de nuestra educación no podrán revertirse.

Haber fungido como asesor de secretarios dio a Pablo Latapí un mayor realismo, pero a la vez lo convenció del valor de lo micro, de lo que ocurre en la escuela, del valor del docente y de su trabajo profesional. El cambio verdadero no lo hace el Estado -el Estado no educa, dice él-. La mejor decisión que puede tomar un secretario de educación es lo que pueda hacer para mejorar la calidad de la formación y la práctica docente.

\section{LA INVESTIGACIÓN SOCIO-PEDAGÓGICA}

La impronta inicial de la investigación que se realizó en el CEE, fundamentalmente por su orientación interdisciplinaria, privi- 
legió la economía y la sociología de la educación. Pablo, como filósofo, logró articular en torno al tema educativo las diversas disciplinas. Su primer artículo periodístico resume su pensamiento inicial al respecto:

La desigualdad económica de la sociedad influye en la desigualdad educativa y se vuelve intergeneracional. A una sociedad de fuertes desigualdades económicas corresponde un sistema escolar de grandes desigualdades educativas. Y mientras el criterio que determine el grado de educación de cada ciudadano sea el nivel económico de su familia, no habrá ni podrá haber justicia social (Latapí, 2012, p. 200).

En su último libro, Finale Prestissimo, expresa esta misma orientación de otra manera:

No es posible lograr una sociedad justa actuando solamente desde la educación. Las decisiones que se toman sobre la educación están fuertemente determinadas por intereses sociales y políticos, de modo que el Estado no es neutral al tomarlas, sino que cede a las presiones de los grupos dominantes. Los grupos o clases sociales de menor poder no pueden hacer valer sus demandas en estas decisiones (Latapí y Quintanilla, 2009, p. 61).

A pesar de ello, se fue haciendo con el tiempo, más y más educador. Se acercó a la pedagogía y escribió con mucha sencillez y claridad para docentes. Ejemplos claros de ello son su incursión en la educación de adultos, la formación valoral y en derechos humanos.

\section{LA EDUCACIÓN DE ADULTOS}

En 1980 se va con María Matilde, su esposa, a una población pequeña en el estado de Querétaro a trabajar en un proyecto de organización campesina y educación popular. Realiza trabajo de alfabetización, lo que lo cimbra profundamente ("El Alfabeto Inútil”). Acepta desde ahí ser investigador de CREFAL. Prolífico como lo era, produce investigaciones y ensayos reunidos en la Antología de este Centro. 


\section{EDUCACIÓN EN DERECHOS HUMANOS}

Desde los inicios de la Asociación Mexicana para las Naciones Unidas, que él fundó, una de las líneas fundamentales del trabajo de la asociación fueron los derechos humanos, y específicamente, la educación en derechos humanos, orientada a docentes en ejercicio en el nivel básico del sistema educativo. Organizó varios diplomados de Educación para la Paz y los Derechos Humanos desde finales de los ochenta, hasta finales de los noventa del siglo pasado. Pablo Latapí tuvo un papel fundamental en la concepción de estos diplomados y en la formación de quienes asumimos la tarea de impartirlos. El fungió también como docente en prácticamente todos ellos.

\section{EL DERECHO A LA EDUCACIÓN}

Si bien el derecho de toda persona a recibir una educación de calidad, y la obligación del Estado de proporcionarla motivó el trabajo de Pablo Latapí a lo largo de su vida, no fue sino hasta su segundo paso por la UNESCO, como embajador, que reconoció la importancia del concepto del Derecho a la Educación. En 2009, en un clásico ensayo publicado en la Revista Mexicana de Investigación Educativa, confiesa este reconocimiento tardío. Entonces no encuentra en México muchos trabajos al respecto, mientras que son patentes grandes violaciones al derecho a la educación en el país y una ausencia casi total de demandas o denuncias al respecto, una situación de "no exigibilidad práctica" de este derecho. Este ensayo se convierte en un llamado a los investigadores educativos a abordar su trabajo desde la perspectiva del Derecho a la Educación, y a los legisladores a tomar medidas para hacer exigible este derecho. Es importante señalar que el INEE asume el reto, y en 2009 presenta su informe anual con el título "El Derecho a la Educación en México".

Habla de que el derecho a la educación es a una educación de calidad. No se cumple o satisface asegurando una escuela o un maestro: tiene que producir los aprendizajes útiles, necesarios, deseados. Y no está de más señalar que la reforma constitucional 
del artículo $3^{\circ}$ dedicado a la educación en 2013 estipula que el Estado garantizará la calidad de la educación obligatoria.

\section{LA EDUCACIÓN EN VALORES Y LA ÉTICA EN LA EDUCACIÓN}

La creciente orientación pedagógica de Pablo Latapí se fue inclinando, como filósofo que era, y orientado por el valor de la justicia, hacia la formación en valores.

En 1996, el entonces secretario de Educación, Miguel Limón Rojas, le pidió que coordinara un grupo de trabajo para proponer la mejor manera de lograr que la escuela básica asumiera su responsabilidad de formación en valores. El fruto del trabajo de este grupo fue la introducción, por primera vez en décadas, de la formación ética en la educación básica. Este trabajo inspiró el bien conocido libro de Pablo Latapí (1999), La moral regresa a la escuela, en el que formula claras reflexiones que le dan carpetazo a la confusión entre el laicismo y la neutralidad valoral de la escuela, y discurre sobre los contenidos de la educación ciudadana, la formación moral y la educación en derechos humanos en el currículo escolar. Este libro, incisivo y crítico como todos sus escritos, pero visionario y propositivo, como el gran educador que era, fue decisivo en otorgar carta de ciudadanía a la educación en derechos humanos en el sistema educativo nacional. Una cita de esta obra clásica:

El Sentido ético es inherente a toda persona. Es una ética de fines. La formación ética no es sometimiento a las normas. La formación moral prepara para elegir, con libertad responsable. La formación moral prepara para afrontar conflictos. La formación en valores conforma la conciencia individual (Latapí, 1999, pp. 20-21).

La preocupación por la ética en la educación lo condujo a formular profundos ensayos reflexivos sobre temas como la tolerancia y el racismo. Sobre la intolerancia, teoriza y propone una pedagogía:

... Interviene la dinámica del conocimiento que, al aceptar algo como verdadero, excluye lo contrario como falso. Hay, dice, una evidente tensión 
entre la legítima y constructiva necesidad de afirmarnos y la ilegítima y destructiva convicción de que somos superiores a los demás (Latapí, 2003, p. 442).

\section{Y luego adopta una clara postura filosófica:}

Me adhiero a una visión ontológica de la persona que fundamenta su especial dignidad y la constituye fin en sí misma, por lo que no puede ser convertida en medio o instrumento de nada (Latapí, 2003, p. 442).

Y la complementa con la visión cristiana de que todos los hombres somos hijos de Dios y, por tanto, hermanos. Sostiene que hay tres zonas de verdades:

La “opinativa”. En esta zona me corresponde relativizar mis opiniones ante las diferentes, dialogar, tratar de enriquecerme. La segunda es la zona cultural, que es valoral. Forman parte de mi visión del mundo y me son irrenunciables. Puedo y debo discutirlos, pero me resulta mucho más difícil relativizarlos. En la tercera zona estarían mis valores absolutos, que son en su mayoría religiosos. No pretendo imponerlos a nadie, pero tampoco les disminuyo el peso y la importancia que para mí tienen, en aras de un pluralismo que todo lo relativizaría. Los que sostienen valores diferentes de la zona dos y tres, ellos tienen derecho a sostenerlas y expresarlas, no porque todas las verdades sean iguales, sino porque ellos tienen la misma dignidad de personas, y la misma libertad de conciencia que yo (Latapí, 2003, p. 442).

De ahí habla de cómo se puede educar para la tolerancia, y sugiere cuatro pasos:

... el primero es trabajar en desmantelar nuestros prejuicios... El segundo es enfrentar al diferente mediante la comunicación y el diálogo. Ante el diferente hay dos maneras incorrectas de proceder: anularlo para afirmarme, o integrarlo a lo mío reduciéndolo a mis categorías, que es otra manera más sutil de anularlo. Lo correcto sería intentar comprenderlo como es y reconocer su propia razón. Un tercer paso es intentar construir juntos algo nuevo a partir de lo mío y de lo que considero valioso en el diferente. Y el cuarto paso es abrirnos a una actitud solidaria con el diferente, lo que 
implica hacer mías sus necesidades y colaborar con él en satisfacerlas. Una convivencia armoniosa supone educarnos en estos estadios progresivos (Latapí, 2003, p. 443).

\section{LOS POBRES, LOS INDÍGENAS}

La justicia social fue su utopía, marcó todas sus búsquedas. Su opción por los pobres está presente en toda su obra.

Leer a México desde los pobres implica acercarse al dolor ajeno como si fuera propio. La opción ética fundamental es la opción por la vida o la opción por la muerte de muchos (Latapí, 2009, p. 144).

Debido a esta opción, la rebelión zapatista lo remueve profundamente. A ella, y a su desenlace, dedica muchos de sus artículos periodísticos en los noventa. Relata cómo descubre a los indígenas de México:

El gran cambio sobrevino en 1994, con el alzamiento zapatista. Vivimos en esa insurrección la posibilidad de que el país virara hacia una perspectiva diferente respecto de su población indígena; que dejáramos de verlos como los más marginados entre los marginados y empezáramos a reconocerles social, jurídica y políticamente su identidad cultural, sus derechos humanos y culturales y un justo espacio de autonomía política... (Latapí, 2009, p. 31).

Nos explica nuestro racismo, y convierte al antirracismo en un propósito educativo necesario:

Se ha considerado a los indígenas como obstáculo para la modernización del país... Está presente la convicción inconfesada de que a causa principal de nuestros defectos se encuentra en nuestro componente indígena. En el fondo nuestro racismo es el rechazo de las culturas indias vivas, pese a la glorificación oficial del indio muerto; la idea que tenemos de país y de su desarrollo excluye a los indios del "nosotros" nacional. No hemos sido capaces de ver nuestra diversidad cultural como una riqueza y de resolver el antagonismo de nuestras culturas constitutivas en un pluralismo aceptado y generoso. La política educativa postrevolucionaria ha ignorado el 
problema de nuestro racismo. Educar para el respeto a la diversidad y la tolerancia debería tener entre nosotros como primer cometido el fomentar en los alumnos una especial sensibilidad hacia los prejuicios raciales (Latapí, 1998, s/p).

Lo llenan la esperanza los Acuerdos de San Andrés, que después serían traicionados:

Si los acuerdos preliminares de San Andrés llegan a plasmarse en reformas legales y se traducen en políticas de Estado, también la educación nacional registrará cambios profundos (Latapí, 1996, s/p.).

\section{PABLO LATAPÍ. LA PERSONA}

Pablo fue una persona muy completa, sumamente versátil, pero siempre un educador. Investigador de la educación. Creador de instituciones, formador de personas. Periodista. Asesor de ministros. Maestro de maestros. Ensayista inspirador.

En su último libro, Finale Prestissimo, Pablo Latapí escribe piezas magistrales de dos o tres páginas cada una, verdaderos ensayos. En ellas nos habla de la política educativa, sí, de los docentes y de la pedagogía también -temas a los que dedicó toda su vida-, pero también de sus valores, personales y religiosos. Nos habla de sus amigos; de su distancia del dinero; de sus representaciones de Dios; del valor educativo de la familia; de la relación que, como un hombre bendecido por una fe profunda e inquebrantable, logró establecer entre esta forma de conocimiento y la razón; de la gratuidad como valor esencial para él; de cómo se acercó a los indígenas, a apreciarlos; de cómo se siente mexicano, su entrega a México, y desde un profundo universalismo multicultural entiende su nacionalismo; de su reflexión epistemológica sobre el misterio; de la importancia que tuvo para él la música; de la prioridad que representan los pobres en su vida, y de la importancia de los valores en la educación. Esta visión integral es la que nos permite conocer al verdadero Pablo Latapí.

Pablo Latapí fue un hombre visionario, un profeta. Generalista en lo educativo -nada de lo educativo le era ajeno- , tuvo en 
el abordaje de múltiples temas una preocupación: la justicia educativa, que en el fondo no es otra cosa que su profundo convencimiento de que la educación es un derecho humano fundamental, clave; que su garante es el Estado, y que para que se cumpla este derecho la educación ha de ser de calidad y debe distribuirse con equidad -a cada quien lo que necesita, y no lo mismo para todos.

Pablo Latapí es un maestro en el pleno sentido de la palabra. Por la calidad de sus trabajos nos conduce a buscar calidad en los nuestros. Por la orientación valoral y comprometida de sus escritos nos entrega un código de ética del investigador educativo que se convierte en un referente central acerca de cómo proceder en nuestra comunidad de investigadores y más allá de ella. Por la congruencia de su vida y sus obras y por la consistencia de su preocupación por la justicia, tanto en sus diversas actividades como a lo largo de su vida, nos presenta una exigencia de congruencia en nuestro propio quehacer. Ésta es la educación que verdaderamente importa.

Pablo Latapí, como todo gran hombre, está vivo. Como toda gran persona, como todo gran clásico, está con nosotros en múltiples momentos de cada día. Difícil escribir en educación sin hacer referencia a alguna obra suya. Imposible dar clases sin mencionarlo. Cuando abate la aflicción y la desesperanza de nuestro país que se desmorona ante la corrupción y la violencia, el que a Pablo le dolía -"me duele México", dijo, en su último discursorecordamos su llamado a la fuerza de la utopía y de la agencia de los seres humanos para lograrla. Le debemos la tarea de redoblar esfuerzos para seguir aplicando sus enseńanzas.

\section{REFERENCIAS BIBLIOGRÁFICAS}

Latapí, P. (1985). "El Alfabeto Inútil", en Proceso, 22 de junio. Latapí, P. (1996). México: la casa de todos sus pueblos. En Proceso, 3 de febrero 1966. [Fecha de consulta: 11 de diciembre de 2018]. Disponible en: https://www.proceso.com. $\mathrm{mx} / 171351 /$ mexico-la-casa-de-todos-sus-pueblos

Latapí, P. (1998). "El racismo, problema educativo nacional". En Proceso, 27 de junio. [Fecha de consulta: 11 de diciem- 
bre de 2018. Disponible en: https://www.proceso.com. $\mathrm{mx} / 178425 /$ el-racismo-problema-educativo-nacional

Latapí, P. (1999). La moral regresa a la escuela. México: IISUEUNAM, Plaza y Valdés.

Latapí, P. (2003) "Raíces de la intolerancia”. En Educere [en línea], 7 (octubre-diciembre): [Fecha de consulta: 19 de noviembre de 2018] Disponible en: http://www.redalyc. org/articulo.oa? id=35602318 $>$ ISSN 1316-4910

Latapí, P. (2008a). Andante con Brío. México: Fondo de Cultura Económica.

Latapí, P. (2008b). Porque ya atardece: algunos textos significativos. México: Edición privada.

Latapí, P. (2009a). Un esfuerzo por construir la educación con personas jóvenes y adultas. Serie Paideia. Pátzcuaro: CREFAL

Latapí, P. (2009b). "El Derecho a la Educación". En Revista Mexicana de Investigación Educativa (14)40, pp. 255-287. Latapí, P. (2012). Educación y Justicia Social. Revista Internacional de Educación para la Justicia Social (RIEJS), 1(1), 199-202.

Latapí, P. y Quintanilla, S. (2009). Finale Prestissimo. Pensamientos, vivencias y testimonios. México: Fondo de Cultura Económica.

Schmelkes, S. (2000). "Una Comunidad de Investigadores". Conferencia de Clausura del V Congreso Nacional de Investigación Educativa. En Consejo Mexicano de Investigación Educativa. V Congreso Nacional de Investigación Educativa: Conferencias Magistrales. México: COMIE. 